\title{
PRÁTICAS DESPORTIVAS NA INFÂNCIA E O IMPACTO NA VIDA ADULTA
}

\author{
Fernanda Varela Büttner e Carolina Machado de Oliveira \\ UNIDAVI \\ nandabuttner@unidavi.edu.br \\ Acesso DOI: http://dx.doi.org/10.34059/ciejop.2019v27i1-17 \\ Resumo
}

A prática esportiva iniciada na infância promove vários benefícios para a saúde da criança, aprimorando e desenvolvendo as capacidades naturais adquiridas com 0 tempo, bem como a socialização, criação de vínculos, amizades, etc. A forma como ocorre esse processo pode alterar de forma significativa o futuro desta criança, bem como sua saúde. Diante disso, o objetivo do presente estudo foi refletir sobre a importância da prática esportiva na infância, em sua relação com os possíveis motivos para o abandono da prática de forma permanente, e o impacto disto na vida adulta. Para a realização desta pesquisa, utilizou-se a base de dados Scielo, Google acadêmico com varredura teórica por meio das palavras-chave: "iniciação esportiva", "saúde" e "abandono", no período de busca entre 1998 a 2018. Os dados analisados indicam que mesmo que uma criança faça parte de uma equipe esportiva, o lúdico deve prevalecer, principalmente para aquelas que praticam determinado esporte somente por lazer e/ou sem fins competitivos. Cabe ao professor/treinador/técnico, instruir cada criança, a fim de proporcionar ganhos na saúde, bem como buscar um equilíbrio entre a recreação e a competição. Práticas desportivas frustradas na infância podem impactar em um adulto sedentário, pois as experiências prévias do mesmo não foram positivas, com isso a culpabilização disso pode recair sobre o profissional. Quanto mais lúdico e divertida for sua experiência dentro da prática, mais fácil se tornará sua permanência na mesma. Quando se trata do público infantil os profissionais de Educação Física que atuam nesse meio, num trabalho multiprofissional com a Psicologia do Esporte, devem levar em consideração o gosto que a criança adquiriu com a prática esportiva, criando estratégias de motivação para a permanência dela no esporte. Com isso, a iniciação esportiva assume um significativo papel de desenvolvimento social, fisiológico e psíquico da criança, tornando a prática esportiva mais prazerosa e saudável.

Palavras chave: Iniciação esportiva; Saúde; Abandono.

\section{Abstract}

The sports practice started in childhood promotes several health benefits for the child, improving and developing the natural abilities acquired over time, as well as socialization, bonding, friendships, etc. The way this process occurs can significantly alter this child's future as well as their health. Therefore, the objective of the present study was to reflect on the importance of sports practice in childhood, its relationship with possible reasons for abandoning the practice permanently, and the impact of this in adult life. In order to carry out this research, we used the Scielo, Google academic database with theoretical scanning through the keywords: "sports 
initiation", "health" and "abandonment", in the search period between 1998 and 2018. The data analyzed indicate that even if a child is part of a sports team, the play should prevail, especially for those who practice a certain sport only for leisure and / or for non competitive purposes. It is up to the teacher / coach / coach to instruct each child in order to provide health gains as well as strive for a balance between recreation and competition. Frustrated sports practices in childhood can impact on a sedentary adult, since the previous experiences of the same were not positive, with that the culpabilization of this can fall on the professional. The more playful and entertaining your experience in practice, the easier your staying will be. When it comes to the children's audience, the Physical Education professionals who work in this environment, in a multiprofessional work with Sports Psychology, must take into account the taste that the child acquired with the practice of sports, creating strategies of motivation for the permanence of it in the sport . With this, sports initiation assumes a significant role of social, physiological and psychic development of the child, making sports practice more pleasurable and healthy.

Keyword: Sports initiation; Cheers; Abandonment

Introdução

Nas últimas décadas, a definição de saúde vem se alterado, isso porque no passado uma pessoa para ser considerada saudável estava associada simplesmente com a ausência de doenças. Nos dias atuais a saúde está mais relacionada com um bem-estar físico, social, espiritual e mental (Dalcagtagne e Lamar, 2012).

A busca pela qualidade de vida vem se tornando cada vez mais significativa, e o esporte está entre as práticas mais procuradas para este estilo de vida, podendo oferecer o alcance de diversos objetivos decorrentes da sua prática (Dalcagtagne; Lamar, 2012).

Os benefícios da atividade física se mantêm em longo período caso a prática seja regular. Se esta prática inicia desde a infância e permanece na vida adulta, melhores resultados poderão ser observados (Bracco et al., 2003).

A maioria dos comportamentos relacionados à pratica esportiva do adulto, é consequente de atitudes similares na infância e adolescência. Isso nos faz pensar que para um adulto estabelecer escolhas saudáveis, a infância e adolescência devem ser as fases mais propícias para o estímulo de uma vida saudável (Romansini, 2007).

Segundo Barbosa et al (1998)

O objetivo principal da prescrição de atividade física na criança e no adolescente é criar o hábito e o interesse pela atividade física, e não treinar visando desempenho. Dessa forma, deve-se priorizar a inclusão da atividade física no cotidiano e valorizar a educação física escolar que Fórum Internacional de Qualidade de Vida e Saúde - Curitiba, 15 de junho de 2019 
estimule a prática de atividade física para toda a vida, de forma agradável e prazerosa, integrando as crianças e não discriminando os menos aptos.

O esporte como fator social é muito ativo na Educação Física, e é uma das formas básicas de preparo das pessoas para a sociedade e para o trabalho, pois envolve a ética, a moral e a ampliação dos laços que podem ser construídos com o passar do tempo (Matveiév, 1986 apud Santos, 2005).

A iniciação esportiva depende de inúmeras razões para que sua prática seja continuada e concretizada. Dentre elas a oportunidade e a acessibilidade fazem parte. A forma como o esporte está inserido dentro da sociedade e como ele é trabalhado para que possa atender a todos que estejam dispostos a praticar, deve ser analisado e se dar uma atenção para esses fatos.

\section{INICIAÇÃO ESPORTIVA}

Quando se fala em iniciação esportiva nota-se a importância de se conhecer o conceito deste termo, pois é de extrema importância entender a sua abrangência para o desenvolvimento da criança (Gabarra et al., 2009).

Segundo Moreira (2003 p.4)

A iniciação esportiva é um marco na vida do ser humano. Dependendo desse primeiro contato, um simples empurrão na piscina, por exemplo, pode levar a traumas, assim como uma base motora construída satisfatoriamente pode gerar segurança. Porém, para que os benefícios aconteçam, esta tem que ocorrer levando em consideração a fase de desenvolvimento do iniciante, pois temos que respeitar a necessidade de experiências para a maturação somática e ainda tomar cuidado com traumas e/ou impactos longitudinais nos membros da criança que está em crescimento, uma vez que, nestas, as cargas intensas no sentido longitudinal predispõem a calcificação das cartilagens de crescimento precocemente.

Vários benefícios são notados com a iniciação esportiva desde a infância, pois contribui para o desenvolvimento físico, social e psicológico da criança, tornando-a assim ativa e saudável, facilitando suas escolhas para se tornar um adulto saudável (Gregório; Silva, 2014).

Diversos estudos com crianças e adolescentes têm demonstrado o benefício da atividade física no estímulo ao crescimento e desenvolvimento, prevenção da obesidade, incremento da massa óssea, aumento da sensibilidade à insulina, melhora do perfil lipídico, diminuição da pressão arterial, desenvolvimento 
da socialização e da capacidade de trabalhar em equipe (Broderick, 2006; Azevedo, 2007 apud Alves; Lima, 2008 p.384).

De acordo com Lazzoli (1998 p. 107) et al.,

Existe associação entre sedentarismo, obesidade e dilipidemias e as crianças obesas provavelmente se tornarão adultos obesos. Dessa forma, criar o hábito de vida ativo na infância e na adolescência poderá reduzir a incidência de obesidade e doenças cardiovasculares na idade adulta. A atividade física também pode exercer outros efeitos benéficos a longo prazo, como aqueles relacionados ao aparelho locomotor.

Para Capitanio (2003) em uma abordagem educativa, o esporte pode ser utilizado como um contexto que disperta o lado crítico da pessoa que o pratica, pois as habilidades técnicas e táticas dão lugar a cooperação, superação e participação, deixando de ser tornar simples praticantes de determinada modalidade esportiva.

Vários métodos são utilizados para se trabalhar com crianças, e o esporte deve ser considerado uma ferramente eficaz nesse sentido, pois além dos benefícios oriundos da prática ele traz valores que podem ser consolidados em outras fases e situações da vida do esportista (Sanches; Rubio, 2011).

Mesmo com vários estudos apontando os benefícios da atividade física na infância e adolescência, poucos jovens são considerados ativos. Apesar de todos os efeitos ligados a saúde, a atividade física quando iniciada na infância pode ser sinônimo de um adulto saudável (Azevedo, 2008 et al., apud Hallal et al, 2010).

\section{ABANDONO DA PRÁTICA ESPORTIVA}

A especialização esportiva precoce é apontada como um grande risco do esporte competitivo durante a iniciação esportiva. A busca incessante pelo prestígio conduz professores e familiares a exporem as crianças a situações de grande exigência e tensão, de treinamentos intensivos e precoces em busca de altos rendimentos (Gabarra et al., 2009 p.5).

Segundo Rubio et al., (2000 apud Fechio et al., 2012) o lúdico passa a se tornar um treinamento visando a técnica, e a recreação dá lugar a competição, quando crianças são inseridas de forma precoce na realidade dos adultos.

O que caracteriza a especialização esportiva são atividades direcionadas a treinamentos especializados. A criança se "especializa" em um determinado esporte visando a técnica de seus fundamentos, além dos 
conhecimentos táticos de apenas um esporte (Whitehead, 1999 apud Fechio et al., 2012).

A saturação esportiva acontece quando a aplicação do modelo de treinamento e competição dos adultos é levada para as crianças. Ao mesmo tempo em que este modelo as atrai pelo alto nível de competição, outras vezes as afasta por fatores como excesso e rigidez de exigências advindas deste tipo de preparação e competição (Todt et al., 2002 apud Fechio et al., 2012).

A falta de prazer foi a principal razão para o abandono do esporte em crianças. Além disso, consequências fisiológicas (lesões no joelho, devido à movimentos repetitivos e intensos) e problemas de ordem psicológica.

Entre os motivos do abandono esportivo, os autores destacam os conflitos de interesses, a falta de tempo, os estudos, a falta de sucesso e de habilidades, o estresse competitivo, a falta de diversão, os treinamentos monótonos, problemas com o treinador, a pequena participação nas competições e as lesões (Butcher et al., 2002 apud Filho; Garcia, 2008 p. 293).

De acordo com Beneli e Montagner (2005), o processo de abadono do esporte no geral se dá devido a vários fatores, dentre eles a competição, onde vencer se torna mais importante que o desenvolvimento em si.

No que diz respeito ao abandono do desporto na adolescência, Gomes (2001, p.34 apud Junior, 2003), afirma que "são várias as causas apontadas, sendo referidas como mais freqüentes as seguintes: a) ter outras coisas para fazer; b) a modalidade não era tão boa como pensava; c) a modalidade não era suficientemente divertida; d) queria praticar outra modalidade; e) sentia-se aborrecido; f) não gostava do treinador; g) o treinador era demasiadamente duro".

Em uma análise geral das múltiplas investigações existentes, se conclui que os motivos para abandonar o esporte são múltiplos e variados, destacando os conflitos de interesse, a falta de tempo, a falta de êxito e de habilidades, o estresse competitivo, a falta de diversão, o desgosto com o treinador, o aborrecimento e as lesões (Junior, 2003).

Ainda Becker Jr. (2000, p.63 apud Junior, 2003) explora que "uma parte importante do abandono é devida à figura do treinador, por gerar estresse competitivo, manter o esportista muito tempo no banco, realizar treinamentos aborrecidos, não motivar e não estimular o suficiente". 


\section{CONCLUSÃO}

Cabe ao professor/treinador/técnico, instruir cada criança, a fim de proporcionar ganhos na saúde, bem como buscar um equilíbrio entre a recreação e a competição. Práticas desportivas frustradas na infância podem impactar em um adulto sedentário, pois as experiências prévias do mesmo não foram positivas, com isso a culpabilização disso pode recair sobre o profissional. Quanto mais lúdico e divertida for sua experiência dentro da prática, mais fácil se tornará sua permanência na mesma.

Quando se trata do público infantil os profissionais de Educação Física que atuam nesse meio, num trabalho multiprofissional com a Psicologia do Esporte, devem levar em consideração o gosto que a criança adquiriu com a prática esportiva, criando estratégias de motivação para a permanência dela no esporte. Com isso, a iniciação esportiva assume um significativo papel de desenvolvimento social, fisiológico e psíquico da criança, tornando a prática esportiva mais prazerosa e saudável.

\section{REFERÊNCIAS}

ALVES, Crésio; LIMA, Renata Villas Boas. Impacto da atividade física e esportes sobre o crescimento e puberdade de crianças e adolescentes. Revista Paulista de Pediatria. São Paulo, v.4, n.26, p. 91-383, 2008.

BARBOSA, Sergio Toledo et al. Atividade física e saúde na infância e adolescência. Rev. Bras. Med. Esporte; vol.4; no;4 Niterói July/Aug. 1998.

BENELI, L.M, MONTAGNER, P.C. Intervenções pedagógicas no processo de evasão do basquetebol: possibilidades e conseqüências. EFDeportes.com, Revista Digital. Buenos Aires, Jul. 2005;10(86).

BRACCO, Mário Maia et al. Atividade Física na infância e adolescência: impacto na saúde pública. Revista de Ciências Médicas. Campinas, v.12, n.1, p. 89-97, jan/mar, 2003.

CAPITANIO, Ana Maria. Educação através da prática esportiva: missão possível? Revista Digital. Buenos Aires, v. 8, n.58, março, 2003.

DALCASTAGNE, Giovanni; LAMAR, Adolfo Ramos. Iniciação esportiva e sua relação com a saúde de crianças e adolescentes. In: SEMINÁRIO DE PESQUISA EM EDUCAÇÃO DA REGIÃO SUL. 5; 2012. Caxias do Sul. Anais. Rio de Janeiro. 
FECHIO, Juliane Jellmayer; CICHOWICZ, Fernanda Duarte Alvez; CASTRO, Natália Martins de; ALVES, Hélio. Especialização esportiva precoce: uma revisão. Revista Digital. Buenos Aires, v. 17, n. 169, jun/2012.

FILHO, Maurício Gattás Bara; GARCIA, Félix Guillén. Motivos do abandono no esporte competitivo: um estudo retrospectivo. Revista Brasileira de Educação Física e Esporte. São Paulo, v.22, n. 4, p. 293-300, out/dez, 2008.

GABARRA, Letícia Macedo; RUBIO, Kátia; ÂNGELO, Luciana Ferreira. A Psicologia do Esporte na iniciação esportiva infantil. Revista Psicologia para América Latina. Buenos Aires, n. 18, nov, 2009.

GREGÓRIO, Karla Mello; SILVA, Thaise da. Iniciação esportiva x especialização esportiva precoce: quando iniciar estas práticas? Horizontes - Revista da Educação. Dourados, n. 3, v. 2, jan/jun, 2014.

HALLAL, Pedro Curi, et al. Prática de Atividade física em adolescentes brasileiros. Revista Ciência e Saúde Coletiva. Rio de Janeiro, v. 15, n.2, p. 30353042, 2010.

JUNIOR, Wilson Antonio Menoncin. Estudos dos fatores que levam os jovens ao abandono da pratica do basquetebol competitivo em Curitiba. 2003. 82f. Dissertação (Mestrado em Engenharia de Produção) - Universidade Federal de Santa Catarina, Florianópolis, 2003.

LAZZOLI, José Kawazoe et al., Atividade física e saúde na infância e adolescência. Revista Brasileira da Medicina do Esporte. São Paulo, v.4, n.4, jul/ago, 1998.

MOREIRA, Sandro Marlos. Pedagogia do esporte e o Karatê-dô: considerações acerca da iniciação e da especialização esportiva precoce. 2003. 233 f. Dissertação (Mestrado em Educação Física) - Faculdade de Educação Física, Universidade Estadual de Campinas, Campinas, 2003.

ROMANSINI, Leandro Augusto. Hábitos de saúde, composição corporal e aptidão física dos participantes da 24ํㅜ edição dos jogos escolares de Santa Catarina. 2007. 135f. Dissertação (Pós-graduação em Ciências do Movimento Humano) - Universidade do Estado de Santa Catarina, Santa Catarina.

SANCHES, Simone Meyer; RUBIO, Kátia. A prática esportiva como ferramenta educacional: trabalhando valores e a resiliência. Revista Educação e Pesquisa. São Paulo, v.37, n.4, p.825-842, dez, 2011. 